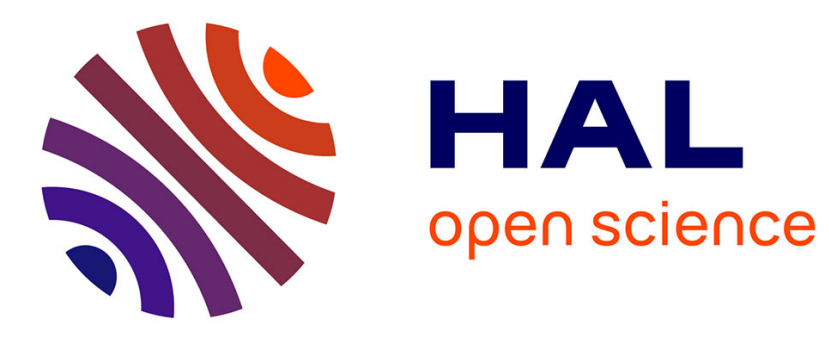

\title{
Simulation of less master production schedule nervousness model
}

Carlos Herrera, André Thomas

\section{To cite this version:}

Carlos Herrera, André Thomas. Simulation of less master production schedule nervousness model. 13th IFAC Symposium on Information Control Problems in Manufacturing, INCOM'2009, Jun 2009, Moscou, Russia. pp.1585-1590, 10.3182/20090603-3-RU-2001.0554 . hal-00511733

\section{HAL Id: hal-00511733 \\ https://hal.science/hal-00511733}

Submitted on 26 Aug 2010

HAL is a multi-disciplinary open access archive for the deposit and dissemination of scientific research documents, whether they are published or not. The documents may come from teaching and research institutions in France or abroad, or from public or private research centers.
L'archive ouverte pluridisciplinaire HAL, est destinée au dépôt et à la diffusion de documents scientifiques de niveau recherche, publiés ou non, émanant des établissements d'enseignement et de recherche français ou étrangers, des laboratoires publics ou privés. 


\title{
Simulation of Less Master Production Schedule Nervousness Model
}

\author{
C. Herrera A. Thomas \\ CRAN, CNRS UMR 7039/Université Henri Poincaré \\ Faculté de Sciences et Techniques, 54506 Vandœuvre-lès-Nancy, France \\ (e-mail: carlos.herrera,andre.thomas@cran.uhp-nancy.fr).
}

\begin{abstract}
In production decision making systems, Master Production Schedule (MPS) states the requirements for individual end items by date and quantity. The solution sensitivity to demand forecast changes, unforeseen supplier and production problem occurrences, is known as nervousness. This feature cause undesirable effects at tactical and operational levels. Some of these effects are production and inventory cost increases and, also, negative impacts on overall and labor productivity. To tackle this problem, we proposed a Mixed Integer Programming (MIP) model where nervousness reduction is carry out finding minimal suboptimal solutions. We perform a simulation under rolling planning horizon environment considering stochastic demand. Results of simulation show that, at each time demand changes take place the model can adjust the solution to achieve less nervousness without a significative change in cost performance.
\end{abstract}

Keywords: Industrial Production Systems, Nervousness, Rescheduling, Mixed Integer Programming.

\section{INTRODUCTION}

In production decision making systems, Master Production Schedule (MPS) states the requirements for individual end items by date and quantity. Moreover, the MPS considers availabilities of critical resources, management policies and goals. The main objective of this plan is to schedule production quantities in each period of the planning horizon, minimizing the cost and maximizing bottleneck utilization. Therefore, the MPS is important to maintain an adequate customer service level and an efficient production system through well managed constraints. The most common way to compute a MPS is to consider a rolling planning horizon, i.e., the plan is constantly computed with a specific periodicity over whole operational horizon. Hence, this methodology regularly reschedules the production quantities by period. The differences in the planned quantities obtained by this rescheduling process, are related to MPS nervousness or instability (our interpretation of these characteristics is proposed in section 2). MPS instability leads to negative effects at both, tactical and operational levels. Some of these effects are production and inventory cost increases and, also, negative impacts on overall and labor productivity (Hayes and Clark, 1985). This changes in the MPS are caused mainly by: end-item forecast changes, lot-sizing rules and scheduled receipts changes (Zhao and Lam, 1997).

When a stability criterion is considered for a MPS, it is necessary to find a trade-off between stability and cost performance (Kimms, 1998), i.e., when the MPS is computed excluding stability, the plan can present huge differences between scheduled quantities in successive computations (cycles). Moreover, this situation makes difficult to apply the plan in practice and leads to huge work in process (WIP), and also, more difficult scheduling (at shop floor level). The most commonly used methods to obtain stable plans are: frozen intervals, planning fences and safety stock. Basically, frozen intervals consist in fixing periods in the planning horizon, in which, changes hoped for later reschedules are not allowed. Planning fences referred to only in certain period changes are not allowed. Finally, safety stock fits inventory levels to absorb uncertainty demand. Nevertheless these methods have a strong static component, because they are based on the idea to fix some variables. On the contrary, the approach presented in this paper searches to reduce the instability by a dynamic process. For this, a mixed integer programming (MIP) model is proposed to compute the MPS, which considers the instability minimization by an implicit way.

The rest of this paper is organized as follows. Section 2 describes the problem. A literature review of some contributions related to MPS stabilization is presented in section 3. In section 4 we describe the classic and proposed MIP formulations of MPS, providing a parametric programming based method to solve this one. Afterward, section 5 explains the design of the simulation experiments and its results are commented. Finally, section 5 presents conclusions and future directions.

\section{PROBLEM DESCRIPTION}

In a rolling planning horizon, MPS computes quantities to produce for a given planning horizon $(n)$, with a specific periodicity $\Delta t$ (cycles). Table 1 shows an example of scheduled quantities resulting of a MPS, where quantity $Q_{k t}$ represents the scheduled production quantity for one end item, for period $t$ obtained by the MPS computed in the cycle $k$. In this example the paremeters are: $n=4$ and $\Delta t=1$. 
To analyze the stability, we make the difference between MPS instability and nervousness. We define MPS instability, as the differences between production quantities scheduled by a MPS in a cycle (e.g., in Table 1 for the cycle $k=2$, differences between $\left.\mathbf{Q}_{\mathbf{2 2}}, \mathbf{Q}_{\mathbf{2 3}}, \mathbf{Q}_{\mathbf{2 4}}, \mathbf{Q}_{\mathbf{2 5}}\right)$. On the other hand, we define nervousness as the difference between quantities scheduled by the MPS in different cycles, for a period in the planning horizon given (i.e. in Table 1 for the period $n=5$, differences between $\mathbf{Q}_{\mathbf{2 5}}$, $\left.\mathrm{Q}_{35}, \mathrm{Q}_{45}, \mathrm{Q}_{55}\right)$.

We define two measures for MPS instability, Mean Instability (MEI) and Maximum Instability (MAI). MEI is defined as:

$$
M E I_{k}^{i}:=\frac{2}{n(n-1)} \sum_{t=k}^{k+n-1} \sum_{t^{\prime}=k+1}^{k+n}\left|x_{t}^{i}-x_{t^{\prime}}^{i}\right|, \quad \forall i, \forall k .
$$

This measure represents the average of the differences of scheduled quantities, between each period and its next periods for all item $i$ and for all cycle $k$.

MAI can be defined as:

$$
\begin{aligned}
& M A I_{k}^{i}:=\max _{t}\left\{\frac{1}{n-t} \sum_{t^{\prime}=k+t}^{k+n}\left|x_{k+t-1}^{i}-x_{t^{\prime}}^{i}\right|,\right. \\
& t=\{1 \ldots, n-1\}, \quad \forall i, \forall k .
\end{aligned}
$$

This measure represents the maximum of the differences of scheduled quantities, between each period and its next periods for all item $i$ and for all cycle $k$.

Now, let $\Omega_{k t}$ the set of overlapped periods in the cycle $k$ for the period $t$, i.e., all scheduled quantities for period $t$ obtained in the precedent cycles. And let,

$$
n_{k}=\sum_{t=k}^{n+k}\left|\Omega_{k t}\right|, \quad \forall k
$$

the set of all overlapped periods for the MPS computed in the cycle $k$, where $|\Omega|$ is the cardinality of the set $\Omega$. Hence,

$$
N A_{k}^{i}=\frac{1}{n_{k}} \sum_{t=k}^{k+n-1} \sum_{h \in \Omega_{k t}}\left|x_{t}^{i}-x_{h}^{i}\right|, \quad \forall i, \forall k,
$$

represents the nervousness computed as the average of the differences of scheduled quantities between all periods in the cycle $k(t=k, k+1, \ldots, k+n-1)$ and all quantities scheduled in precedent cycles (the last period are not overlapped periods), for all item $i$ and for all cycle $k$. This measure will be referred as Nervousness All Periods (NA).

Another measure defined is the nervousness for the first period. This measure represents the nervousness computed by similar way as above but, only considering the first period. This is considered because, if $\Delta t=1$ hence the

Table 1. Example of MPS in a rolling horizon

\begin{tabular}{ccccccccc}
\hline $\mathrm{k} / \mathrm{t}$ & 1 & 2 & 3 & 4 & 5 & 6 & 7 & 8 \\
\hline 1 & $Q_{11}$ & $Q_{12}$ & $Q_{13}$ & $Q_{14}$ & & & & \\
2 & & $\mathbf{Q}_{\mathbf{2 2}}$ & $\mathbf{Q}_{\mathbf{2 3}}$ & $\mathbf{Q}_{\mathbf{2 4}}$ & $\mathbf{Q}_{\mathbf{2 5}}$ & & & \\
3 & & & $Q_{33}$ & $Q_{34}$ & $\mathbf{Q}_{35}$ & $Q_{36}$ & & \\
4 & & & & $Q_{44}$ & $\mathbf{Q}_{45}$ & $Q_{46}$ & $Q_{47}$ & \\
5 & & & & & $\mathbf{Q}_{55}$ & $Q_{56}$ & $Q_{57}$ & $Q_{58}$ \\
\hline
\end{tabular}

implemented period is only the first period. This measure is defined as:

$$
N F_{k}^{i}=\frac{1}{n_{k f}} \sum_{h \in \Omega_{k f}}\left|x_{k}^{i}-x_{h}^{i}\right|, \quad \forall i, \forall k
$$

where $n_{k f}$ represents the number of overlapped periods in the cycle $k$ only for the first period in the MPS. This measure will be referred as Nervousness First Period (NF).

Nervousness has a dynamic character because it takes place when the differences of the computed quantities in different cycles are obtained.

\section{LITERATURE REVIEW}

Many works study MPS stability and nervousness effects and their impact on production planning and control systems. In the following some contributions related to MPS stabilization are presented.

Inderfurth (1994) studies the nervousness effect for stochastic inventory control. For that, he defines a measure of nervousness taking into account exclusively the setup variable. The results show that, nervousness is affected by the control rules. Following this work, the instability measures defined in the previous subsection, can be classified as long-term stability and quantity-oriented. This means that, we consider the whole planning horizon for the computations of the differences between production quantities.

In the work of Kadipasaoglu and Sridharan (1995), the following strategies to reduce nervousness are studied: freezing the MPS, end-item safety stock and lot-for-lot lotsizing rule for components. Some factors considered are: item cost structure, items structure, level of demand uncertainty and lot-sizing method. Decision variables are: cost, instability, and customer service level. The main result is that, frozen periods strategy, presents the best performance under stochastic demand multilevel environment.

In Zhao and Lam (1997) the effects on the stability of the plans produced by the interaction between several lotsizing rules and frozen interval selection are studied. The study is performed with a simulation and a completely randomized full factorial design, in order to test the different hypotheses. The results show a strong dependency between MPS instability and lot-sizing rules as well as frozen interval selection. Some works make a similar study for different lot-sizing problems, see Xie et al. (2003, 2004).

From the perspective of production planning models, Kimms (1998) explains the impact of the stability in three problems of production planning. Also, some approaches are proposed to measure stability, applying them to the MPS. The effects for different cost structures are simulated and analyzed. Finally, an iterative method is proposed to solve MPS.

Kazan et al. (2000) propose three methods to reduce MPS instability. The first two methods correspond to modified versions of classic Wagner-Within and SilverMeal methods. Finally, a method based on MIP that takes in consideration previous scheduled periods to solve MPS is proposed. The results are analyzed by simulation, where, 
the model based on mixed integer programming, obtains good stability results in some cases.

For a scheduling problem Rangsaritratsamee et al. (2004) considers jointly the optimization of efficiency and stability measures. In the first part, the inclusion of the stability as a variable to be optimized is justified. To solve the problem, an approach of genetic algorithm is proposed. Finally, the results are analyzed by statistical methods concluding that, the stability does not affect drastically the production system efficiency.

Analyzing the effects of the system structure on the stability, in the context of the supply chain, Meixell (2005) studies a model for lot-sizing multi-level multiitem problem and considers structural variables as: setup cost, relationships between components and capacity. The statistical results show a strong relation between the considered variables and stability. The results of this work highlight that it is possible to take emphasize in the following fact: "fewer production orders and capacity increase have stabilizing effects on the plans". Following this line, van Donselaar et al. (2000) study the impact of material coordination concepts in supply chain stability, with a more practical approach. Their results identify three variables that affect strongly the stability, theses are: lot-sizes, level of uncertainty in demand and items structure.

The works of Richter and Sombrutzki (2000) and Tang and Grubbström (2002) express the importance of considering stability in MRP systems in the context of remanufacturing process and MPS frozen interval selection.

Finally, Thomas et al. (2008) propose a mathematical programming method to obtain a stable MPS. This approach is developed with a two steps model at tactical level. In the aggregated plan (S\&OP), is proposed a procedure called reference plan, that considers compromises between successive planning periods to reduce changes. At detailed plan level (MPS), a heuristic procedure is proposed to obtain a stable MPS. The main result of this work is to improve the stability of the detailed plan.

\section{LESS MASTER PRODUCTION SCHEDULE INSTABILITY FORMULATION}

In the first part of this section, we present the classic MIP formulation for a MPS $\left(\mathcal{F}_{1}\right)$, which is considered as a benchmark and our proposed formulation $\left(\mathcal{F}_{2}\right)$ also.

\subsection{Formulation}

Formally, a mixed integer programming formulation for a MPS, considering $m$ items, $n$ periods and $r$ resources, can be stated as follows:

\section{Variables}

$$
\begin{array}{lll}
x_{t}^{i} & : & \text { production of item } i \text { in period } t . \\
s_{t}^{i} & : & \text { inventory of item } i \text { in period } t . \\
y_{t}^{i} & : & \text { setup variable for item } i \text { in period } t, \text { where } \\
y_{t}^{i}= & \left\{\begin{array}{l}
1 \text { if } x_{t}^{i}>0 \\
0 \text { if } x_{t}^{i}=0
\end{array}\right.
\end{array}
$$

\section{Parameters}

$d_{t}^{i} \quad: \quad$ demand of item $i$ in period $t$.

$\bar{p}_{t}^{i} \quad$ : $\quad$ production cost of item $i$ in period $t$.

$h_{t}^{i} \quad: \quad$ inventory cost of item $i$ in period $t$.

$q_{t}^{i} \quad$ : $\quad$ setup cost of item $i$ in period $t$.

$L_{t}^{r} \quad$ : available capacity of resource $r$ in period $t$.

$\alpha^{i r} \quad$ : unitary consumption of resource $r$ by production of item $i$.

$\beta^{i r} \quad$ : setup time for item $i$ on resource $r$.

$M_{t}^{i}=\sum_{t=1}^{n} d_{t}^{i}, \quad \forall i$, upper bound of production of item $i$ in period $t$.

\section{Formulation $\mathcal{F}_{1}$}

$$
\begin{gathered}
\min \sum_{i=1}^{m} \sum_{t=1}^{n}\left(\bar{p}_{t}^{i} x_{t}^{i}+h_{t}^{i} s_{t}^{i}+q_{t}^{i} y_{t}^{i}\right) \\
s_{t-1}^{i}+x_{t}^{i}=d_{t}^{i}+s_{t}^{i}, \quad \forall i, t \\
x_{t}^{i} \leq M_{t}^{i} y_{t}^{i}, \quad \forall i, t \\
\sum_{i=1}^{m}\left(\alpha^{i r} x_{t}^{i}+\beta^{i r} y_{t}^{i}\right) \leq L_{t}^{r}, \quad \forall r, t \\
x \in \mathbb{R}_{+}^{m n}, s \in \mathbb{R}_{+}^{m(n+1)}, y \in\{0,1\}^{m n} .
\end{gathered}
$$

The objective function (6) searches to minimize the costs of production, inventory and setup. Constraint (7) represents the inventory balance and constraint (8) the relationship between production and setup. The constraint (9) represents the available capacity of resources by period. This formulation can be found in Pochet and Wolsey (2006) and will be referred as $\mathcal{F}_{1}$. The objective function value of this formulation will be referred as $z\left(\mathcal{F}_{1}\right)$.

\section{Formulation $\mathcal{F}_{2}$}

Without loss of generality, we assume $s_{0}=0$, and using:

$$
s_{t}^{i}=\sum_{l=1}^{t}\left(x_{l}^{i}-d_{l}^{i}\right), \quad \forall i, \quad 1 \leq l \leq n,
$$

now we can to replace inventory variable $s_{t}^{i}$ in the objective function (6) and to obtain:

$$
\min \sum_{i=1}^{m} \sum_{t=1}^{n}\left(p_{t}^{i} x_{t}^{i}+q_{t}^{i} y_{t}^{i}\right)-\sum_{i=1}^{m} \sum_{t=1}^{n} h_{t}^{i} d_{t}^{i},
$$

where the new production cost (inventory included) is:

$$
p_{t}^{i}=\bar{p}_{t}^{i}+\sum_{j=t}^{n} h_{j}^{i}, \quad \forall t, i .
$$

The constant term $\sum_{i=1}^{m} \sum_{t=1}^{n} h_{t}^{i} d_{t}^{i}$ can be eliminated of the objective function. In Brahimi et al. (2006) this formulation is showed for the single-item lot-sizing problem (SILSP). In our case the above formulation is applied to capacitated multi-item lot-sizing problem (CMILSP). A new 
formulation without inventory variables also considering stabilization of the MPS, can be expressed as follows:

$$
\begin{gathered}
\min \sum_{i=1}^{m} \sum_{t=1}^{n}\left(p_{t}^{i} x_{t}^{i}+q_{t}^{i} y_{t}^{i}\right)+\sum_{i=1}^{m} \sum_{t=1}^{n-1}\left(\lambda^{i} \omega_{t}^{i}\right) \\
\sum_{j=1}^{t} x_{j}^{i} \geq D_{t}^{i}, \quad \forall i, \forall t \\
x_{t}^{i}-x_{t+1}^{i} \leq \omega_{t}^{i}, \quad \forall i \quad \text { and } \quad 1 \leq t \leq n-1 \\
x_{t+1}^{i}-x_{t}^{i} \leq \omega_{t}^{i}, \quad \forall i \quad \text { and } \quad 1 \leq t \leq n-1 \\
x_{t}^{i} \leq M_{t}^{i} y_{t}^{i}, \quad \forall i, \forall t \\
\sum_{i=1}^{m}\left(\alpha^{i r} x_{t}^{i}+\beta^{i r} y_{t}^{i}\right) \leq L_{t}^{r}, \quad \forall r, t \\
x \in \mathbb{R}_{+}^{m n}, \omega \in \mathbb{R}_{+}^{m(n-1)}, y \in\{0,1\}^{m n} .
\end{gathered}
$$

The objective function (14) searches to minimize the costs of production, inventory and setup, and also, the differences between consecutive production quantities (in the same cycle). In this formulation constraint (15) express that cumulative production in any period for each item must be equal or greater than the cumulative demand $D_{t}^{i}$ in the same period (backlog is not allowed). This formulation will be referred as $\mathcal{F}_{2}$. The new objective value noted $Z\left(\mathcal{F}_{2}\right)$ can be obtained computing the inventory variable $s_{t}^{i}$ from (11) using the optimal solutions computed by $\mathcal{F}_{2}$. Note that in $\mathcal{F}_{2}$ we are only interested in the new optimal solutions $x_{t}^{i}$ for a specific value of $\lambda^{i}$ (for the concerned cycle) to obtain a new objective function value from (6). Moreover note that to compute $\mathcal{F}_{2}$ with $\lambda^{i}=0, \forall i$, is equivalent to compute $\mathcal{F}_{1}$.

The variable $\omega_{t}^{i}$ represents an auxiliary variable to minimize the quantity differences between consecutive periods in whole planning horizon $n$. The parameter $\lambda^{i}$ represents a control parameter (defined by user) for item instability minimization. This formulation can be viewed as equivalent to minimize $\lambda^{i}\left|x_{t+1}^{i}-x_{t}^{i}\right|, \forall i$ and $1 \leq t \leq n-1$.

Note that, in the measures (1) and (2) it is necessary to compute the differences between all periods in a specific cycle. This is equivalent to consider ${ }_{n} C_{2}$ quantity difference combinations. Nevertheless, considering the high computational cost of this, the minimization is performed only in one step, i.e., for a given production quantity, the model searches to minimize the difference between its antecessor and successor values only.

Moreover, no explicit measure for nervousness minimization have been considered in the model. The reduction of nervousness is considered to be implicit in the instability reduction.

\section{SIMULATION}

\subsection{Experimental design}

The results are obtained for a simulation horizon of $H=$ 52 weeks, with rescheduling interval $\Delta t=1$ and for a planning horizon $n=8$.

For each cycle $k=1,2, \ldots, 52$, we solve the model $\mathcal{F}_{2}$ for $\lambda=0,1,2, \ldots$. Supposing that $\delta$ is the maximum deviation from the optimal cost allowed, hence we compute $\mathcal{F}_{2}$ until total cost not exceeds this value. For the simulation we chose an arbitrary $\delta=5 \%$.

The parameters $p_{t}^{i}, h_{t}^{i}, q_{t}^{i}$ are randomly generated and uniformly distributed. The demand for each period is randomly generated as follows:

$$
d_{1 j}^{i} \sim U\left(d f^{i}, d l^{i}\right), \quad \forall i \quad \text { and } \quad 1 \leq j \leq n .
$$

This represents the demand for the first cycle, where $U\left(d f^{i}, d l^{i}\right)$ is the uniform distribution between parameters $d f^{i}$ and $d l^{i}$, for each item $i$. The demand quantities for the following planning cycles are obtained as:

$$
d_{k j}^{i}=d_{(k-1) j}^{i}+\epsilon_{j}, \quad \forall i \quad \text { and } \quad k \leq j \leq k+n-1,
$$

where,

$$
\epsilon_{j} \sim U[0, c j], \quad k \leq j \leq k+n-1, \quad c \in \mathbb{N} .
$$

Parameter $\epsilon_{j}^{i}$ represents a random error for period $j$ and item $i$, and $c$ is used to control the error variance. The demand quantities for the new last periods are generated as:

$$
d_{k(k+n)}^{i} \sim U\left(d f^{i}, d l^{i}\right)
$$

The capacity parameter $L_{t}^{r}$ must be consistent with constraint (7) and (15) for the formulations $\mathcal{F}_{1}$ and $\mathcal{F}_{2}$ respectively, to insure feasibility (Pochet and Wolsey, 2008). Hence this parameter is randomly generated such that the following condition holds,

$$
\sum_{i=1}^{m}\left(\alpha^{i r} d_{t}^{i}+\beta^{i r}\right) \leq L_{t}^{r}, \quad \forall r, t .
$$

Furthermore, $r=1$ is considered, supposing this resource as a fixed bottleneck of the system. Table 2 summarize the simulation parameters.

Table 2. Simulation parameters

\begin{tabular}{cc}
\hline Parameter & Value \\
\hline Items & 2 \\
Planning horizon & 8 \\
Operational horizon & 52 \\
Demand $(\mathrm{d})$ & $\mathrm{U}[100,140]$ \\
Production $(p)$ & $\mathrm{U}([95,105])$ \\
Inventory $(h)$ & $\mathrm{U}([95,105])$ \\
Setup $(q)$ & $\mathrm{U}([10000,20000])$ \\
$r$ & 1 \\
Capacity $(L)$ & 150 \\
$\alpha$ & $\mathrm{U}[0.01,0.02]$ \\
$\beta$ & $\mathrm{U}[2,3]$ \\
$c$ & 1 \\
\hline
\end{tabular}

Simulation code was developed in Python 2.5. ${ }^{1}$. The MIP programs were solved with Glpk $4.29^{2}$.

\subsection{Results}

Figure 1 highlights that for the $\lambda_{\max }$ value (the most expensive and the most stable choice) we obtain a drastic instability reduction (about $60 \%$ ). Note that probably the decision maker prefers to choice $\lambda_{d}$ for a best compromise, i.e., not too high cost increasing and significative instability reduction.

\footnotetext{
1 http://www.python.org

2 http://www.gnu.org/software/glpk/
} 


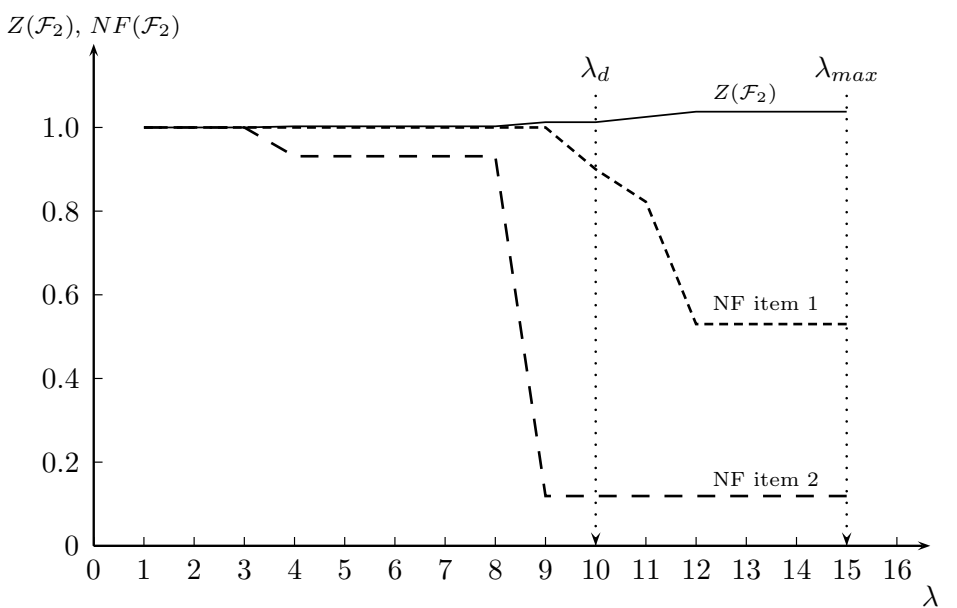

Fig. 1. Results of $Z\left(\mathcal{F}_{2}\right)$ and $N F\left(\mathcal{F}_{2}\right)$ varying $\lambda$ at cycle 9

In table 3 column $Z\left(\mathcal{F}_{2}\right)$ shows the augmented cost and columns $N F\left(\mathcal{F}_{2}\right), N A\left(\mathcal{F}_{2}\right)$ the reduced values of nervousness with respect to $\mathcal{F}_{1}$. These values are obtained using $\lambda_{\max }(\delta=5 \%)$ for the two items considered. We observe that although the cost does not increase more than $5 \%$ $N F$ and $N A$ have and important instability reduction.

\section{CONCLUSIONS}

In this paper we perform a simulation of a mixed integer programming model for Master Production Schedule taking into account the minimization of MPS nervousness. The results show that this formulation leads to reduce MPS nervousness considerably without a great difference in terms of total cost compared with a classical formulation.

In future works others decision rules will be studied leading to more robust solutions and muffling nervousness.

\section{REFERENCES}

Brahimi, N., Dauzere-Peres, S., Najid, N., and Nordli, A. (2006). Single item lot sizing problems. European Journal of Operations Research, 168(1), 1-16.

Hayes, R. and Clark, K. (1985). Explaining observed productivity differentials between plants: Implications for operations research. Interfaces, 15(6), 3-14.

Inderfurth, K. (1994). Nervousness in inventory control: analytical results. OR Spektrum, 16, 113-123.

Kadipasaoglu, S. and Sridharan, V. (1995). Alternative approaches for reducing schedule instability in multistage manufacturing under demand uncertainty. Journal of Operations Management, 13, 193-211.

Kazan, O., Nagi, R., and Rump, C. (2000). New lotsizing formulations for less nervous production schedules. Computers \& Operations Research, 27(13), 13251345 .

Kimms, A. (1998). Stability measures for rolling schedules with applications to capacity expansion planning, master production scheduling, and lot sizing. Omega, 26(3), $355-366$.

Meixell, M. (2005). The impact of setup costs, commonality, and capacity on schedule stability: An exploratory study. International Journal of Production Economics, 95(1), 95-107.
Table 3. Results of cost and nervousness for $\lambda_{\max }$

\begin{tabular}{|c|c|c|c|c|c|}
\hline \multirow[b]{2}{*}{ Cycle } & \multirow[b]{2}{*}{$Z\left(\mathcal{F}_{2}\right)$} & \multicolumn{2}{|c|}{$N F\left(\mathcal{F}_{2}\right)$} & \multicolumn{2}{|c|}{$N A\left(\mathcal{F}_{2}\right)$} \\
\hline & & I1 & $\mathrm{I} 2$ & I1 & $\mathrm{I} 2$ \\
\hline 2 & 1,04 & 0,44 & 0,42 & 1,00 & 0,97 \\
\hline 3 & 1,05 & 0,15 & 0,22 & 0,25 & 1,00 \\
\hline 4 & 1,04 & 0,28 & 0,51 & 1,00 & 1,00 \\
\hline 5 & 1,04 & 0,40 & 0,11 & 1,00 & 1,00 \\
\hline 6 & 1,02 & 0,52 & 0,20 & 1,00 & 1,00 \\
\hline 7 & 1,05 & 0,07 & 0,19 & 0,02 & 1,00 \\
\hline 8 & 1,02 & 0,48 & 0,15 & 1,00 & 1,00 \\
\hline 9 & 1,04 & 0,53 & 0,12 & 1,00 & 1,00 \\
\hline 10 & 1,04 & 0,10 & 0,09 & 1,00 & 0,24 \\
\hline 11 & 1,03 & 0,07 & 0,40 & 0,31 & 1,00 \\
\hline 12 & 1,03 & 0,21 & 0,38 & 1,00 & 1,00 \\
\hline 13 & 1,05 & 0,12 & 0,39 & 0,31 & 1,00 \\
\hline 14 & 1,04 & 0,47 & 0,11 & 1,00 & 0,44 \\
\hline 15 & 1,03 & 0,66 & 0,39 & 1,00 & 1,00 \\
\hline 16 & 1,05 & 0,55 & 0,15 & 1,00 & 0,41 \\
\hline 17 & 1,04 & 0,38 & 0,32 & 0,38 & 1,00 \\
\hline 18 & 1,04 & 0,64 & 0,49 & 1,00 & 1,00 \\
\hline 19 & 1,05 & 0,10 & 0,08 & 0,43 & 1,00 \\
\hline 20 & 1,04 & 0,26 & 0,06 & 1,00 & 0,45 \\
\hline 21 & 1,04 & 0,29 & 0,03 & 0,44 & 1,00 \\
\hline 22 & 1,03 & 0,73 & 0,06 & 1,00 & 0,43 \\
\hline 23 & 1,03 & 0,57 & 0,15 & 1,00 & 0,41 \\
\hline 24 & 1,03 & 0,56 & 0,03 & 1,00 & 0,43 \\
\hline 25 & 1,04 & 0,09 & 0,05 & 0,45 & 1,00 \\
\hline 26 & 1,04 & 0,32 & 0,04 & 1,00 & 0,45 \\
\hline 27 & 1,05 & 0,36 & 0,05 & 0,45 & 1,00 \\
\hline 28 & 1,04 & 0,07 & 0,67 & 0,43 & 1,00 \\
\hline 29 & 1,04 & 0,07 & 0,16 & 0,44 & 1,00 \\
\hline 30 & 1,03 & 0,55 & 0,22 & 1,00 & 1,00 \\
\hline 31 & 1,03 & 0,11 & 0,25 & 0,44 & 0,41 \\
\hline 32 & 1,04 & 0,12 & 0,20 & 0,48 & 1,00 \\
\hline 33 & 1,04 & 0,26 & 0,16 & 0,42 & 1,00 \\
\hline 34 & 1,02 & 0,38 & 0,56 & 0,51 & 1,00 \\
\hline 35 & 1,04 & 0,15 & 0,20 & 1,00 & 0,43 \\
\hline 36 & 1,04 & 0,11 & 0,21 & 1,00 & 0,47 \\
\hline 37 & 1,03 & 0,57 & 0,76 & 1,00 & 1,00 \\
\hline 38 & 1,05 & 0,04 & 0,51 & 0,46 & 1,00 \\
\hline 39 & 1,04 & 0,10 & 0,50 & 1,00 & 0,42 \\
\hline 40 & 1,03 & 0,69 & 0,51 & 1,00 & 0,49 \\
\hline 41 & 1,04 & 0,18 & 0,69 & 1,00 & 1,00 \\
\hline 42 & 1,04 & 0,14 & 0,65 & 1,00 & 1,00 \\
\hline 43 & 1,05 & 0,11 & 0,63 & 0,42 & 1,00 \\
\hline 44 & 1,04 & 0,18 & 0,18 & 1,00 & 0,50 \\
\hline 45 & 1,02 & 0,25 & 1,00 & 0,41 & 1,00 \\
\hline 46 & 1,05 & 0,42 & 0,24 & 1,00 & 1,00 \\
\hline 47 & 1,04 & 0,45 & 0,18 & 1,00 & 0,43 \\
\hline 48 & 1,03 & 0,27 & 0,48 & 0,48 & 1,00 \\
\hline 49 & 1,05 & 0,23 & 0,48 & 0,43 & 1,00 \\
\hline 50 & 1,04 & 0,39 & 0,41 & 1,00 & 0,51 \\
\hline 51 & 1,05 & 0,38 & 0,14 & 1,00 & 1,00 \\
\hline
\end{tabular}

Pochet, Y. and Wolsey, L. (2006). Production planning by mixed integer programming. Springer New York, New York.

Pochet, Y. and Wolsey, L. (2008). Single item lot-sizing with non-decreasing capacities. Mathematical Programming Serie A.

Rangsaritratsamee, R., Jr., W.F., and Kurz, M.B. (2004). Dynamic rescheduling that simultaneously considers efficiency and stability. Computers $\mathscr{G}$ Industrial Engineering, 46(1), 1-15.

Richter, K. and Sombrutzki, M. (2000). Remanufacturing planning for the reverse wagner/whitin models. European Journal of Operations Research, 121(2), 304-315. 
Tang, O. and Grubbström, R. (2002). Planning and replanning the master production schedule under demand uncertainty. International Journal of Production Economics, 78(3), 323-334.

Thomas, A., Genin, P., and Lamouri, S. (2008). Mathematical programming approaches for stable tactical and operational planning in supply chain and aps context. Journal of Decision Systems, 17, 425-455.

van Donselaar, K., van den Nieuwenhof, J., and Visschers, J. (2000). The impact of material coordination concepts on planning stability in supply chains. International Journal of Production Economics, 68(1), 169-176.

Xie, J., Lee, T., and Zhao, X. (2004). Impact of forecasting error on the performance of capacitated multi-item production systems. Computers $\mathscr{B}$ Industrial Engineering, $46(2), 205-219$.

Xie, J., Zhao, X., and Lee, T. (2003). Freezing the master production schedule under single resource constraint and demand uncertainty. International Journal of Production Economics, 83(1), 65-84.

Zhao, X. and Lam, K. (1997). Lot-sizing rules and freezing the master production schedule in material requirements planning systems. International Journal of Production Economics, 53(3), 281-305. 